\title{
Syndecan-4 as a biomarker to predict clinical outcome for glioblastoma multiforme treated with WT1 peptide vaccine
}

\begin{abstract}
Aim: In cancer immunotherapy, biomarkers are important for identification of responsive patients. This study was aimed to find biomarkers that predict clinical outcome of WT1 peptide vaccination. Materials \& methods: Candidate genes that were expressed differentially between long- and short-term survivors were identified by CDNA microarray analysis of peripheral blood mononuclear cells that were extracted from 30 glioblastoma patients (discovery set) prior to vaccination and validated by quantitative RT-PCR using discovery set and different 23 patients (validation set). Results: SDC-4 mRNA expression levels distinguished between the long- and short-term survivors: 1-year survival rates were 64.0 and $18.5 \%$ in SDC4-low and -high patients, respectively. Conclusion: SDC-4 is a novel predictive biomarker for the efficacy of WT1 peptide vaccine.
\end{abstract}

Lay abstract: Recently, cancer immunotherapies are becoming a standard therapeutic option. To improve their efficacy, identification of biomarkers is important to select responsive patients. In this study, we identified $S D C-4$ as a biomarker to predict clinical outcome using peripheral blood mononuclear cells obtained from patients with glioblastoma, a malignant brain tumor, who were treated with WT1 peptide vaccine. With 30 samples of peripheral blood mononuclear cells prior to vaccination, 32 candidate genes were filtrated by microarray, and finally only SDC-4 was validated by RT-PCR using another 23 samples. Accordingly, 1-year survival rates were 64.0 and $18.5 \%$ in SDC4-low and -high patients, respectively.

First draft submitted: 9 November 2015; Accepted for publication: 4 January 2016; Published online: 3 October 2016

Keywords: cancer immunotherapy $\bullet \mathrm{DC}-\mathrm{HIL} \bullet \mathrm{GBM} \bullet$ glioblastoma multiforme $\bullet$ immune checkpoint inhibitor $\bullet$ malignant glioma $\bullet$ peptide vaccine $\bullet$ SDC-4 $\bullet$ Syndecan-4 $\bullet$ WT1

WT1, a transcription factor, regulates many kinds of important genes that play important roles in embryogenesis, cell proliferation and differentiation [1-3]. The WT1 gene is overexpressed in leukemia and a variety of solid tumors, in which it exerts an oncogenic function $[4,5]$. WT1, a pan-tumor-associated antigen (TAA), was identified as the best one among 75 TAAs, based on criteria including therapeutic function, immunogenicity, oncogenicity, specificity, expression levels and percent of positive cells, expression levels in stem cell, number of patients with antigenpositive cancers, number of epitopes and cellular localization [6]. Our group and others have performed WT1-targeted cancer immunotherapy, including WT1 peptide vaccination, and WT1 peptide-pulsed [7] or WT1 mRNA-electroporated [7-9] dendritic cell (DC) therapy, and obtained a series of successful results with positive immunological and clinical responses in patients with glioblastoma multiforme (GBM) [10-14], acute myeloid leukemia [15-19], chronic myeloid leu-
Satoshi Takashima', Yoshihiro Oka ${ }^{1,2,3}$, Fumihiro Fujiki², Soyoko Morimoto², Hiroko Nakajima², Yoshiki Nakae ${ }^{1,4}$, Jun Nakata Sumiyuki Nishida', Naoki Hosen $^{6}$, Naoya Tatsumi ${ }^{5}$, Kenji Mizuguchi', Naoya Hashimoto 8,9 , Yusuke Oji6, Akihiro Tsuboi ${ }^{5}$, Atsushi Kumanogoh ${ }^{1,3}$ \& Haruo Sugiyama*,2

*Author for correspondence: Department of Cancer Immunology, Osaka University Graduate School of Medicine, 1-7 Yamadaoka, Suita City, Osaka 565-0871, Japan Tel. \& Fax: +81 668792593 sugiyama@sahs.med.osaka-u.ac.jp For full list of affiliations, please see back page 
kemia [20,21], myelodysplastic syndromes [22-24], multiple myeloma [25], malignant melanoma [26], infantile rhabdomyosarcoma [27], and lung [28], breast [15], pancreatic $[29,30]$, ovarian $[31,32]$, uterine $[23,33]$ and salivary gland cancers [34,35].

GBM is a malignant brain tumor with very poor prognosis. The standard therapy for the newly diagnosed GBM is surgery, followed by irradiation and chemotherapy. However, a 5-year survival rate is less than $10 \%$, and once recurrence occurs, therapeutic options are limited [36]. In 2008, we reported promising results from a Phase II clinical study of WT1 peptide vaccination in patients with recurrent or conventional therapy-resistant GBM. In patients who received the vaccination, progression-free survival (PFS) at 6 months was $33.3 \%$, and the median overall survival (OS) was 36.7 weeks, suggesting the therapeutic potential of WT1 peptide vaccine for GBM patients [10].

In order to improve the clinical usefulness of WT1 peptide vaccine, it is crucial not only to biologically enhance the vaccine's efficacy but also to select patients who are likely to respond to the vaccine. Identification of responders would be facilitated by the availability of reliable biomarkers that predicted the clinical outcome of patients treated with the WT1 peptide vaccine.

To date, several studies by our group and others have identified markers that are correlated with the clinical efficacy of WT1-based immunotherapy. Malignant glioma patients with higher WT1 expression levels (score 3-4) lived longer after immunotherapy than those with lower expression levels (score 1-2) [13]. An increase in the frequency of WT1-specific cytotoxic $\mathrm{T}$ lymphocytes (CTLs) before and after WT1 peptide vaccination was correlated with clinical response $[15,17,30]$. Upon repeated vaccination with WT1 peptide, an increase in the frequencies of effector memory subsets, which are important for maintenance of WT1-specific CTLs, was also identified as a predictor of responders [30]. Lymphocyte numbers in peripheral blood and antigen-specific delayed-type hypersensitivity (DTH) also predict clinical outcome in various immunotherapies [37]. However, no reliable biomarkers have yet been established that can predict the clinical outcome of immunotherapies, such as TAA-targeting cancer vaccines prior to therapy. High-throughput technologies, such as cDNA microarray analysis, have been investigated as approaches to discover biomarkers for prediction of clinical outcomes of therapeutic interventions.

In this study, we used cDNA microarrays to comprehensively analyze gene-expression profiles of peripheral blood mononuclear cells (PBMCs) with the goal of identifying biomarkers that predicted the outcome of WT1 peptide vaccination in patients with recurrent or conventional therapy-resistant GBM. The results revealed that $S D C-4$ mRNA expression levels prior to WT1 vaccination were a promising predictive biomarker for clinical outcome in these patients.

\section{Materials \& methods}

\section{WT1 peptide vaccine}

The WT1 peptide vaccine consists of WT1-CTL epitope peptide and Montanide ISA51 adjuvant. The peptide used in this study is a modified 9-mer WT1 peptide (amino acids [aa] 235-243 CYTWNQMNL; mWT1-235), in which $\mathrm{M}$ was replaced by $\mathrm{Y}$ at the second amino acid position, an anchor position for $H L A-A^{*} 24: 02$, of the natural WT1-235 peptide (235-243 CMTWNQMNL; nWT1-235). The binding affinity of mWT1-235 for HLA-A*24:02 is higher than that of nWT1-235, and mWT1-235 induced a much stronger CTL response against WT1-expressing tumor cells. GMP-grade WT1 peptide was purchased from Multiple Peptide Systems (CA, USA) and Peptide Institute (Osaka, Japan) as the lyophilized peptide.

\section{Patients}

Sixty patients were enrolled in a Phase II study of WT1 peptide vaccination of GBM patients, the result of which we reported previously $[10,13]$. Patients with recurrent or progressive GBM were eligible to be enrolled in the Phase II study if their disease was resistant to conventional chemotherapy or radiotherapy. Other inclusion criteria were as described in our previous study. Briefly, they were: first, age between 16 and 80 years; second, expression of WT1 in glioma cells as determined by immunohistochemical analysis; third, HLA-A*2402-positivity; fourth, estimated survival of more than 3 months; fifth, ECOG Performance Status Grade $0-2$; sixth, no severe organ function impairment and seventh, written informed consent of the patient. All enrolled patients were histologically proven to have GBM (Grade 4) based on the WHO criteria.

PBMC samples obtained prior to WT1 vaccination were available from 53 of the 60 patients enrolled in the Phase II study; all 53 were used in this study. Thirty of the patients were randomly assigned to the discovery set, and the remaining 23 were assigned to the validation set.

The median OS of the patients in the discovery set was 347 days from the start of the vaccination and 460 days from the time of recurrence or disease progression to death or censored time points. The median PFS, which was defined as the days from the start of the vaccination to the disease progression, was 62 days. In the microarray analysis, to select differentially expressed genes (DEGs) that influence not only short-term outcome (RECIST, PFS and OS from the time of vac- 
cination started) but also long-term outcome, the 30 patients in a discovery set were divided into two groups with OS from the time of recurrence or disease progression. The 15 patients with $O S$ of $\geq 460$ days were defined as long-term survivors (OS median: 1133 days, range: 480-2678 days), whereas the remaining 15 patients with OS of $<460$ days were defined as shortterm survivors (OS median: 216 days, range: 138-458 days) (Table 1).

In the verification process using discovery set, OS was the days from the first vaccination to death or censored time points which should more strictly reflect the vaccination effect. Thus, in the verification step, patients who survived $\geq 347$ days (the median OS of the discovery set according to the immediately preceding definition) were defined as long-term survivors, whereas those who survived $<340$ days were defined as short-term survivors.

In the validation set, median OS from the first vaccination was 257 days. The 12 patients who survived $\geq 256$ days were defined as long-term survivors, and the 11 patients who survived $<256$ days were defined as short-term survivors.

All short-term survivors in the discovery and validation sets died before a censored time point. Therefore, patient categorization of long- and short-term survivors was fixed (Supplementary Table 1). The Phase II study of WT1 peptide vaccination and cDNA microarray analysis of the blood samples was approved by the ethical review board of Osaka University Hospital.

\section{WT1 peptide vaccination schedule}

After informed consent was obtained, weekly intradermal injection of $3.0 \mathrm{mg}$ of HLA-A*24:02-restricted
mWT1-235 peptide emulsified with Montanide ISA51 adjuvant was initiated. Vaccinations were scheduled for 12 consecutive weeks, after which responses were evaluated by MRI. Responses were classified as complete response, partial response, stable disease and progressive disease using MRI according to the RECIST (Response Evaluation Criteria In Solid Tumors) criteria. When clinical response was obvious, WT1 vaccination was continued at 2-week intervals for the next few months, and then at 1-3-month intervals until obvious tumor progression or deterioration of the patient's condition was observed.

\section{Blood samples}

Peripheral blood was obtained from patients immediately before the first vaccination, followed by separation of PBMCs by density gradient centrifugation using Lymphocyte Separation Solution (Nacalai Tesque, Kyoto, Japan). Separated PBMCs were stored in liquid nitrogen prior to use.

\section{RNA isolation from PBMCs \& cDNA microarray analysis}

Frozen PBMCs were thawed, and total RNA was isolated using the TRIzol reagent (Life Technologies, CA, USA), purified using chloroform and subjected to isopropanol and ethanol precipitation. Purified total RNA was quantitated using a NanoDrop ND-1000 (Thermo Fisher Scientific, MA, USA).

RNAs from PBMCs of the discovery set patients were sent to Toray Industries (Tokyo, Japan), which performed RNA-based cDNA microarray analysis using Human oligo chip 25k ver1.00 (Toray Industries).

Table 1. Patients' characteristics in a discovery set.

\begin{tabular}{|c|c|c|c|c|c|}
\hline \multirow[t]{2}{*}{ Characteristic } & \multirow[t]{2}{*}{ Short OS $(n=15)$} & \multirow[t]{2}{*}{ Long OS $(n=15)$} & \multicolumn{2}{|c|}{ Hazard ratio $95 \% \mathrm{Cl}$} & \multirow[t]{2}{*}{ p-value } \\
\hline & & & Lower & Upper & \\
\hline \multicolumn{6}{|l|}{ Age (years): } \\
\hline - Mean (range) & $58(25-67)$ & $46(20-75)$ & 0.07 & 2.76 & 0.36 \\
\hline \multicolumn{6}{|l|}{ Gender: } \\
\hline - Male (\%) & $9(60)$ & $11(73)$ & 0.35 & 3.88 & 0.93 \\
\hline \multicolumn{6}{|l|}{ KPS: } \\
\hline - Mean (range) & $75(45-95)$ & $80(55-100)$ & 0.005 & 0.50 & $0.009 *$ \\
\hline Surgical treatment (\%) & $13(87)$ & $15(100)$ & 0.15 & 11.7 & 0.90 \\
\hline Chemotherapy, n (\%) & $14(93)$ & $11(73)$ & 1.26 & 26.5 & $0.021 *$ \\
\hline \multicolumn{6}{|c|}{ RT before vaccination (Gy): } \\
\hline - Average (SD) & $67(22.3)$ & $61(8.7)$ & 0.03 & 13.3 & 0.96 \\
\hline
\end{tabular}


(A)

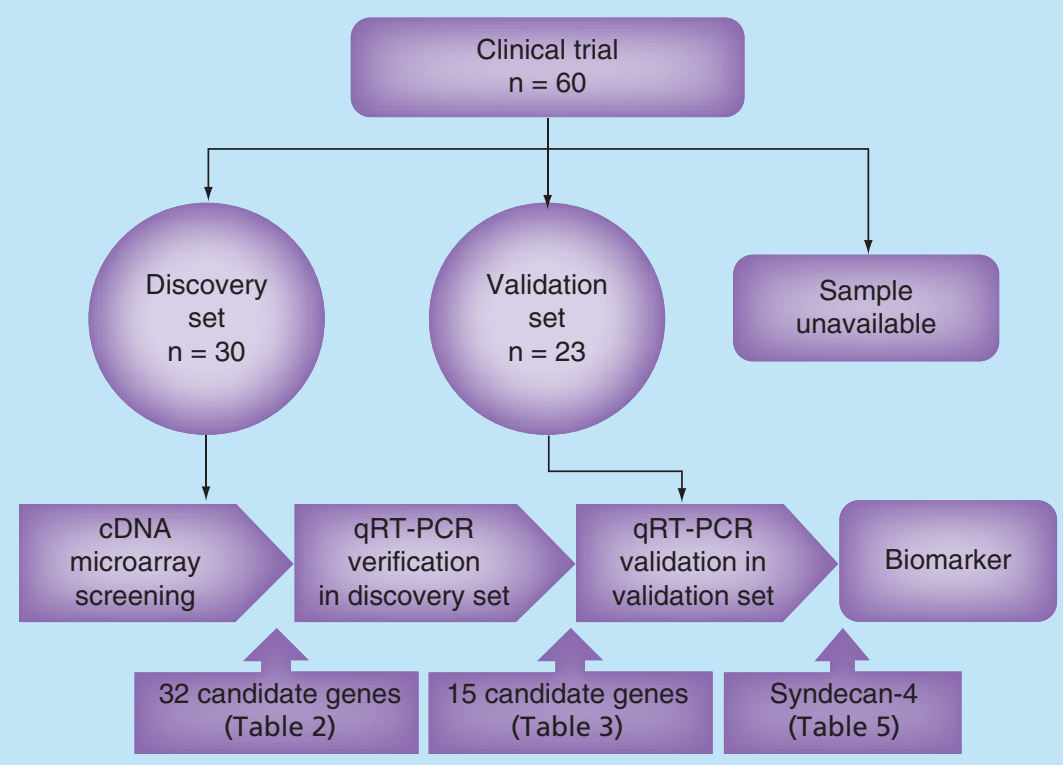

(B)

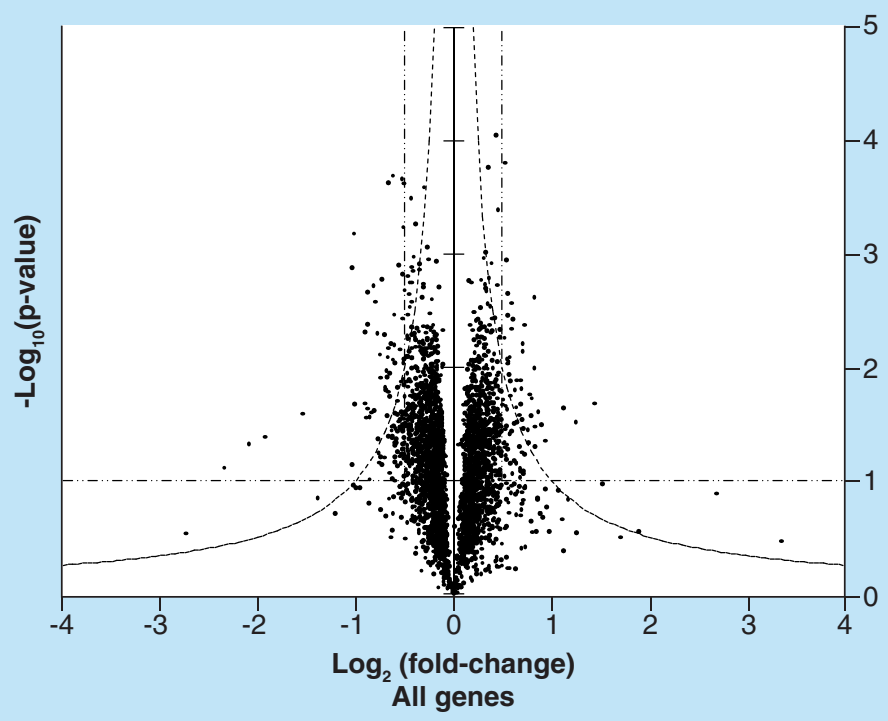

(C)

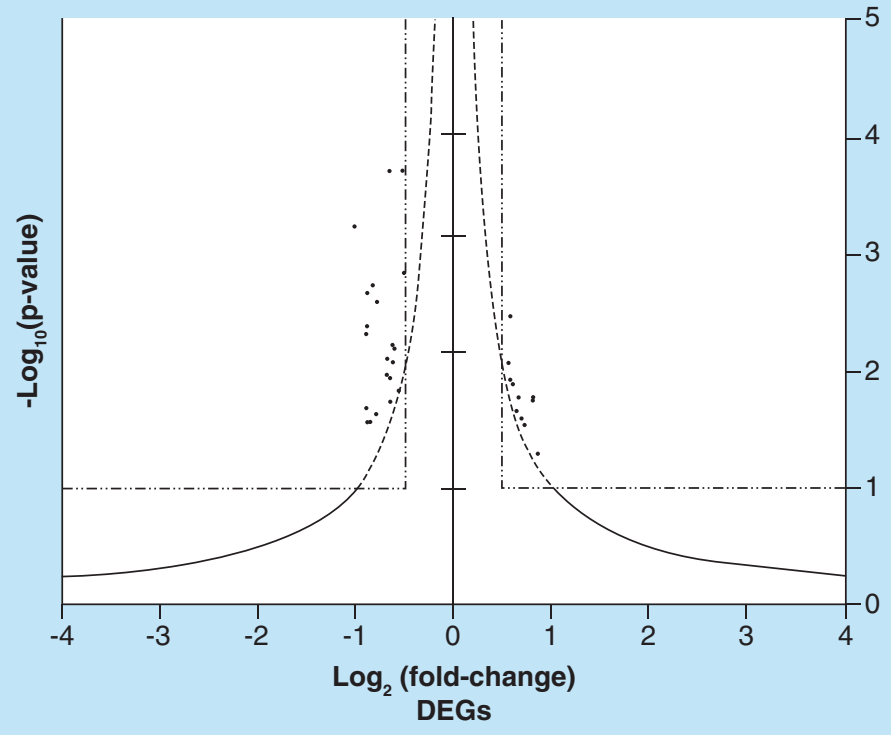


Figure 1. Selection of candidate genes by CDNA microarray analysis (see facing page). (A) Strategy to find biomarkers is schematically shown. First, DEGs were screened by CDNA microarray and the expression levels of screened genes were verified by quantitative RT-PCR using 30 glioblastoma multiforme patients in a discovery set. Second, these verified DEGs were validated using different 23 GBM patients in a validation set. Finally, only SDC-4 was identified as a biomarker. (B) A volcano plot was generated. Each dot corresponds to one gene. X-and $y$-axes indicate fold change ( $\log _{2}[$ short/long]) of signal intensities of individual genes and the statistical significance $\left(-\log _{10}[p\right.$-value $\left.]\right)$ of the difference in the signal intensities of individual genes between long- and short-term survivors, respectively. Dashed line indicates $y=|x|^{-1}, y=1$ and $|x|=0.5$. (C) Thirty-two candidate DEGs were extracted as described in the text. Gene names and their statistical evaluations are shown in Table 2.

DEG: Differentially expressed gene.

Microarray analysis data were transformed using global normalization, followed by quantile normalization [38]. First, genes with intensities less than ([average intensities of blank spots] $+10 \times$ [standard deviation of intensities of blank spots]) were excluded. To identify DEGs between long- and short-term survivors, a volcano plot of $-\log _{10}$ (p-value) of Welch's t-test between the two groups (y-axis) versus $\log _{2}$ (fold change) (x-axis) was made [39]. The fold change between the two groups was calculated as $\log _{2}$ ([mean signal intensities in long-term survivors]/[mean signal intensities in short-term survivors]). DEGs were selected using this volcano plot according to the following conditions: $y$ $>|\mathrm{x}|^{-1},|\mathrm{x}|>0.5$ and $\mathrm{y}>1.0$. To enrich for DEGs, correlations between individual gene expression intensities and either PFS or clinical responses according to RECIST criteria were examined by Pearson's and Spearman's correlation coefficient, respectively. Genes that did not exhibit significant p-values (alpha level 0.1 for Pearson's correlation coefficient and 0.2 for Spearman's correlation coefficient) were excluded from further analysis. In this series of microarray analyses, a nonstringent cut-off for the $\mathrm{p}$-value was set according to MAQC guidelines [40]. Finally, genes that satisfied the following six conditions were retained for further analysis: first, average intensity $\geq$ ([average intensities of blank spots] $+10 \times$ [standard deviation of intensities of blank spots]); second, $-\log _{10}$ (p-value of difference between long- and short-OS groups) was greater than $\mid \log _{2}$ (fold difference) $\left.\right|^{-1}$; third, $\mid \log _{2}$ (fold difference) $\mid$ was greater than 0.5 ; fourth, $-\log _{10}$ (p-value of difference between long- and short-OS groups) greater than 1.0; fifth, $\mathrm{p}$-value of Pearson's correlation between PFS and signal intensities less than 0.1 ; and sixth, $\mathrm{p}$-value of Spearman's correlation between clinical outcome and signal intensities less than 0.2 .

\section{RT-PCR}

RNAs from PBMCs were reverse-transcribed into first-strand cDNA using SuperScript VILO cDNA Synthesis Kit and Master Mix (Life Technologies). Sequences of primers used for RT-PCR are provided in Supplementary Table 2. Target sequences are available from the NCBI nucleotide database [41]. Sample cDNAs were preamplified with dilute primer mixture for 15 cycles prior to loading to the BioMark 48.48 dynamic array nanofluidic chips (Fluidigm, CA, USA). RT-PCR was performed on a BioMark HD (Fluidigm). Regarding internal control genes, we quantified many control genes, including $A C T B, G A P D H$, RPL13, RPL18A, PPIA, TSR2 and RNA28S1. Each control gene was validated in comparison with each other, and correlation matrix was generated, followed by scoring each gene. Consequently, ACTB was determined as the most stable internal control gene among all the candidate control genes and was used as the internal control. Then, $\triangle \mathrm{CT}$ s were calculated as ([CT value of each genes] - [CT value of $A C T B]$ ). All data concerning gene expression levels were converted to $\log _{2}\left(1+2^{-\Delta C T}\right)$, and the relative expression levels were statistically analyzed.

\section{Statistical analysis}

Cox proportional hazard regression models were used to evaluate associations between patient characteristics and OS in the discovery and validation sets. In the microarray analysis, PFS was logarithmically transformed to yield a normal distribution.

In statistical analysis of the result from RT-PCR, the Jarque-Bera test was used to assess normality of variable distributions, and the F-test was used to test homogeneity of variance.

To promote statistical power, parametric analyses were conducted as much as possible. For examination of the difference between long- and short-term survivors, an appropriate transformation (e.g., logarithmic, square-root, cube-root or fourth-root transformation) was performed for individual genes to satisfy the requirement for normality. All normally distributed variables were checked for homogeneity of variance and analyzed by Student's t-test. Non-normally distributed variables were analyzed using the Mann-Whitney U-test.

The SDC-4 expression levels that discriminated responders and nonresponders (SDC-4 cut-off value) were determined using a receiver operating characteristic (ROC) curve in order to maximize the Youden's index (defined as Youden's J statistic = sensitivity + specificity -1). The ROC curve was drawn based on the cut-off value of OS, which maximized area under the ROC curve (AUC). The horizontal and vertical 
axes indicate 1-specificity and sensitivity for responder, respectively. Sensitivity, specificity, positive and negative predictive values, and accuracy of the prediction of OS by the SDC-4 expression levels were calculated using standard formulas. Kaplan-Meier curves and a two-sided log-rank test were used to assess differences between two groups defined by the SDC-4 cut-off value. Statistical analysis was conducted using appro- priate software including JMP Pro 10.0 (SAS Institute, Inc., NC, USA) and R-commander [42].

\section{Results}

Identification of DEGs in PBMCs prior to WT1 vaccination

Patients with recurrent or conventional therapyresistant GBM who were treated with WT1 peptide

\begin{tabular}{|c|c|c|}
\hline Gene & Fold change $^{\dagger}$ & p-value ${ }^{\ddagger}$ \\
\hline TLR10 & 0.85 & 0.051 \\
\hline$K L H D C 8 B$ & 0.80 & 0.018 \\
\hline RALGPS2 & 0.80 & 0.017 \\
\hline CD79B & 0.71 & 0.029 \\
\hline TNFAIP8L2 & 0.69 & 0.026 \\
\hline GCNT2 & 0.65 & 0.017 \\
\hline EVA1 & 0.64 & 0.022 \\
\hline CETN3 & 0.59 & 0.013 \\
\hline ROGDI & 0.58 & 0.012 \\
\hline CD82 & 0.57 & 0.004 \\
\hline IL17RA & 0.55 & 0.009 \\
\hline TNFSF14 & -1.02 & $<0.001$ \\
\hline CST1 & -0.90 & 0.005 \\
\hline FBXO32 & -0.90 & 0.021 \\
\hline ULBP2 & -0.88 & 0.028 \\
\hline$O A S L$ & -0.88 & 0.004 \\
\hline PHLDA1 & -0.88 & 0.002 \\
\hline FASLG & -0.86 & 0.028 \\
\hline XAGE5 & -0.83 & 0.002 \\
\hline$S D C 4$ & -0.80 & 0.024 \\
\hline ZNF659 & -0.79 & 0.003 \\
\hline SMAD7 & -0.69 & 0.011 \\
\hline SLC7A5 & -0.68 & 0.008 \\
\hline VPS37B & -0.66 & $<0.001$ \\
\hline$H P G D$ & -0.65 & 0.018 \\
\hline MLF1 & -0.65 & 0.012 \\
\hline$S K I$ & -0.63 & 0.006 \\
\hline EXPH5 & -0.63 & 0.009 \\
\hline ZC3H12A & -0.61 & 0.007 \\
\hline UAP1 & -0.57 & 0.015 \\
\hline CCNT1 & -0.53 & $<0.001$ \\
\hline ITGA5 & -0.52 & 0.002 \\
\hline
\end{tabular}




\begin{tabular}{|c|c|c|c|c|c|c|}
\hline Gene & Transformation & $\begin{array}{l}\text { Normal } \\
\text { distribution }\end{array}$ & Statistical method & $\begin{array}{l}\text { Fold } \\
\text { change }^{\dagger}\end{array}$ & $p$-value & Result \\
\hline EXPH5 & Square root & True & Left-sided Student t-test & -0.34 & $<0.001 *$ & Verified \\
\hline ZC3H12a & Failed & False & $\begin{array}{l}\text { Left-sided Mann-Whitney } \\
\text { U test }\end{array}$ & -1.23 & $0.004 *$ & Verified \\
\hline VPS37B & Not needed & True & Left-sided Student t-test & -1.19 & $0.005^{*}$ & Verified \\
\hline PHLDA1 & Square root & True & Left-sided Student t-test & -1.33 & $0.007^{*}$ & Verified \\
\hline TNFSF14 & 4th root & True & Left-sided Student t-test & -1.70 & $0.007^{*}$ & Verified \\
\hline UAP1 & Square root & True & Left-sided Student t-test & -1.58 & $0.013 *$ & Verified \\
\hline HPGD & Cube root & True & Left-sided Student t-test & -0.68 & $0.016^{*}$ & Verified \\
\hline$S K I$ & 4 th root & True & Left-sided Student t-test & -0.76 & $0.016^{*}$ & Verified \\
\hline$S D C 4$ & Logarithmic & True & Left-sided Student t-test & -0.35 & $0.023 *$ & Verified \\
\hline FOX032 & Logarithmic & True & Left-sided Student t-test & -2.02 & $0.023^{*}$ & Verified \\
\hline SLC7A5 & Square root & True & Left-sided Student t-test & -0.97 & $0.026^{*}$ & Verified \\
\hline ULBP2 & Logarithmic & True & Left-sided Student t-test & -2.04 & $0.030 *$ & Verified \\
\hline CCNT1 & Cube root & True & Left-sided Student t-test & -0.60 & $0.031 *$ & Verified \\
\hline FASLG & Cube root & True & Left-sided Student t-test & -1.46 & $0.036 *$ & Verified \\
\hline OASL & Square root & True & Left-sided Student t-test & -1.04 & $0.039 *$ & Verified \\
\hline SMAD7 & Square root & True & Left-sided Student t-test & -1.53 & 0.067 & Excluded \\
\hline GCNT2 & Failed & False & $\begin{array}{l}\text { Right-sided Mann-Whitney } \\
\text { U-test }\end{array}$ & 3.82 & 0.189 & Excluded \\
\hline EVA1 & Not needed & True & Right-sided Student t-test & 0.00 & 0.220 & Excluded \\
\hline ITGA5 & Cube root & True & Left-sided Student t-test & -0.76 & 0.241 & Excluded \\
\hline F3 & Failed & False & $\begin{array}{l}\text { Left-sided Mann-Whitney } \\
\text { U-test }\end{array}$ & -2.11 & 0.252 & Excluded \\
\hline XAGE5 & Failed & False & $\begin{array}{l}\text { Left-sided Mann-Whitney } \\
\text { U-test }\end{array}$ & -1.77 & 0.261 & Excluded \\
\hline MLF1 & Square root & True & Left-sided Student t-test & -0.46 & 0.448 & Excluded \\
\hline TLR10 & Failed & False & $\begin{array}{l}\text { Right-sided Mann-Whitney } \\
\text { U-test }\end{array}$ & 4.73 & 0.491 & Excluded \\
\hline CETN3 & Cube root & True & Right-sided Student t-test & 0.05 & 0.523 & Excluded \\
\hline CD82 & Square root & True & Right-sided Student t-test & -0.08 & 0.577 & Excluded \\
\hline TNFAIP8L2 & Cube root & True & Right-sided Student t-test & -0.40 & 0.646 & Excluded \\
\hline$C D 79 B$ & Square root & True & Right-sided Student t-test & 0.45 & 0.653 & Excluded \\
\hline IL17RA & Cube root & True & Right-sided Student t-test & 0.47 & 0.738 & Excluded \\
\hline RALGPS2 & Not needed & True & Right-sided Student t-test & -0.20 & 0.744 & Excluded \\
\hline$K L H D C 8 B$ & Logarithmic & True & Right-sided Student t-test & 0.37 & 0.821 & Excluded \\
\hline ZNF659 & Failed & False & $\begin{array}{l}\text { Left-sided Mann-Whitney } \\
\text { U-test }\end{array}$ & 1.42 & 0.934 & Excluded \\
\hline ROGD1 & Square root & True & Right-sided Student t-test & 0.07 & 0.982 & Excluded \\
\hline
\end{tabular}

vaccine were selected as the cohort for this analysis. PBMCs were obtained prior to WT1 vaccination from 53 of 60 patients in this vaccination trial. The strategy to search useful biomarkers for prediction of clinical outcome is shown schematically in Figure 1A. Patients were assigned randomly into the discovery and vali- 
dation sets (30 and 23 patients, respectively); within the validation set, patients were classified as long-term or short-term survivors (15 patients each) (Table 1). Cox proportional hazard regression models revealed that within the discovery set, there was no association between OS following vaccination and age, gender, presence or absence of prior surgical treatment, or prior total absorbed dose of radiotherapy; however, low-performance status and history of chemotherapy were indicators of worse clinical outcome (Table 1). We discussed these issues that affect clinical outcome later in the last part of the Results and Discussion section.

To identify candidate genes that were expressed differentially between long- and short-term survivors, we performed cDNA microarray analysis on PBMCs. A volcano plot of statistical significance $\left(-\log _{10}\right.$ p-value) versus $\log _{2}$ (fold change), in which each dot indicates one of a total of 25,000 genes, is shown in Figure 1B. DEGs were extracted as follows. First, we excluded genes with intensities less than ([average intensities of blank spots] $+10 \times$ [standard deviation of intensities of blank spots]). The remaining 3037 genes were filtrated to a set of 74 genes that satisfied the following three criteria, $y>|x|^{-1},|x|>0.5$ and $y>1.0$. To further enrich for candidate genes, the correlation between the signal intensities of individual genes and either PFS or clinical response was evaluated by Pearson's correlation and Spearman's rank correlation analysis, respectively, and genes with p-values $\geq 0.1$ (Pearson's) or $\geq 0.2$ (Spearman's) were excluded. Ultimately, 32 genes were selected as candidates (Table 2 \& Figure 1C). Of these candidate genes, 11 were highly expressed in long-term survivors (Table 2, upper); and 21 were highly expressed in short-term survivors (Table 2, lower).
Verification of candidate genes by quantitative RT-PCR

Expression levels of the 32 candidate genes identified in the cDNA microarray analysis of the patients in the discovery set were verified by quantitative RT-PCR.

In order to ensure a rigorous statistical analysis, individual gene expression levels obtained by quantitative RT-PCR were transformed appropriately to satisfy the requirement for normality. Distributions were checked for normality by the Jarque-Bera test, for homogeneity of variance by the F-test, and for significance by one-tailed Student's t-test (Table 3). When no transformation yielded a sufficiently normal distribution, the one-tailed Mann-Whitney U-test was used. Because one-tailed tests were used, genes with fold changes that were inverted relative to those obtained from the microarrays were automatically excluded. As a result of these analyses, 15 genes whose expression levels correlated to OS were retained, and 17 were excluded (Table 3).

\section{Verification of 15 candidate genes using} patients in the validation set

Next, we prepared a validation set consisting of 23 GBM patients treated with WT1 peptide vaccine following the same protocol used in the discovery set. Characteristics of patients in the validation set are provided in Table 4. No association was detected between OS and age, gender, performance status, the presence or absence of prior surgical treatment and chemotherapy, or prior total absorbed doses on radiotherapy. Using data from the patients in the validation set, we investigated whether the expression levels of the 15 candidate genes identified in the discovery set correlated with OS using the same statistical methods as those used in the discovery set. Only expression of

Table 4. Patients' characteristics in a validation set.

\begin{tabular}{|c|c|c|c|c|c|}
\hline \multirow[t]{2}{*}{ Characteristic } & \multirow[t]{2}{*}{ Short OS $(n=11)$} & \multirow[t]{2}{*}{ Long OS $(n=12)$} & \multicolumn{2}{|c|}{ Hazard ratio $95 \% \mathrm{Cl}$} & \multirow[t]{2}{*}{ p-value ${ }^{\dagger}$} \\
\hline & & & Lower & Upper & \\
\hline \multicolumn{6}{|l|}{ Age (years): } \\
\hline - Mean (range) & $48.9(28-63)$ & $50.8(30-71)$ & 0.14 & 5.48 & 0.90 \\
\hline \multicolumn{6}{|l|}{ Gender: } \\
\hline - Male (\%) & $7(58)$ & $7(63)$ & 0.21 & 1.88 & 0.40 \\
\hline \multicolumn{6}{|l|}{ KPS: } \\
\hline - Mean (range) & $78(50-100)$ & $84(50-100)$ & 0.08 & 2.21 & 0.29 \\
\hline Surgical treatment (\%) & $10(91)$ & $12(100)$ & 0.06 & 19.9 & 0.86 \\
\hline Chemotherapy, No. (\%) & $9(82)$ & $10(83)$ & 0.11 & 2.12 & 0.28 \\
\hline \multicolumn{6}{|c|}{ RT before vaccination (Gy): } \\
\hline - Average (SD) & $57(9.0)$ & $59(2.9)$ & 0.005 & 2.54 & 0.13 \\
\hline
\end{tabular}


Table 5. Validation of 15 candidate genes using different 23 glioblastoma multiforme patients in a validation set.

\begin{tabular}{|llll|}
\hline Gene & Fold change $^{\dagger}$ & p-value & Result \\
\hline SDC4 & -1.85 & $0.020^{*}$ & Validated \\
\hline HPGD & -0.84 & 0.076 & Not reproducible \\
\hline PHLDA1 & -0.59 & 0.154 & Not reproducible \\
VPS37B & -1.81 & 0.269 & Not reproducible \\
\hline OASL & -0.21 & 0.485 & Not reproducible \\
\hline TNFSF14 & 0.27 & 0.555 & Not reproducible \\
\hline FASLG & 3.62 & 0.602 & Not reproducible \\
\hline UAP1 & 2.75 & 0.608 & Not reproducible \\
\hline SLC7A5 & 1.82 & 0.679 & Not reproducible \\
\hline FOXO32 & 2.21 & 0.760 & Not reproducible \\
\hline SKI & 3.80 & 0.844 & Not reproducible \\
\hline EXPH5 & 2.43 & 0.869 & Not reproducible \\
\hline ULBP2 & 2.65 & 0.953 & Not reproducible \\
\hline ZC3H12a & 2.71 & 0.964 & Not reproducible \\
\hline CCNT1 & 1.09 & 0.975 & Not reproducible \\
\hline${ }^{\circ}$ Log ${ }_{2}$ (long/short). & & \\
*Statistical significance $(p<0.05)$. & & \\
\hline
\end{tabular}

SDC-4 significantly (negatively) correlated with OS in the validation set (Table 5).

\section{Prognostic prediction of GBM patients} treated with WT1 peptide vaccine using SDC-4 expression levels

First, based on the values of OS and $S D C-4$ expression levels, we generated a ROC curve using all 53 patients from the discovery and validation sets (Figure 2A). Generally, area under the ROC curve (AUC) of an efficient biomarker shows high AUC, maximum of which is 1.0. Since AUC varies in accordance with cut-off values of OS, the cut-off value was selected in order to maximize the AUC; therefore, the patients were classified into two groups, 'responders' and 'nonresponders' by cut-off value of 256 days and then AUC was 0.72 . The best cut-off value of $S D C$ - 4 expression levels to classify responders (OS of $\geq 256$ days) and nonresponders (OS of <256 days) was determined to be 0.001 according to the AUC-maximized ROC curve using the Youden's index; SDC-4 expression levels $\leq 0.001$ and $>0.001$ predicted responders and nonresponders, respectively. Prediction of likely responders had $70.4 \%$ sensitivity, $76.0 \%$ specificity, $76.0 \%$ positive prediction value, $70.4 \%$ negative prediction value and $73.1 \%$ accuracy; in addition, a Chi-square test demonstrated a significant difference $(\mathrm{p}<0.001)$.

Furthermore, we performed survival analysis using this cut-off value of $S D C-4$ expression level (0.001).
The patients were divided into two groups, $S D C$ 4 -low $(S D C-4 \leq 0.001)$ and -high $(S D C-4>0.001)$ groups (Figure 2B). The difference in OS between the two groups was estimated by Kaplan-Meier curves and the subsequent log-rank test. The results revealed that $S D C$-4-low patients survived significantly longer than $S D C$-4-high patients; 1-year OS rates were 64.0 and $18.5 \%$, respectively (Figure $2 \mathrm{~B}$ ).

As shown in Table 1, low-performance status and the past history of chemotherapy were indicated to be risk factors of worse clinical outcome for the discovery set patients. Therefore, potential confounding effects of SDC-4 expression levels and baseline patient characteristics, including the two factors mentioned above, were assessed using Cox proportional hazard regression models in all the 53 patients (Supplementary Table 3). This analysis allowed us to assess the association between $S D C-4$ expression levels and the risk of death at any given time points while controlling for other predictors that may affect the risk of death. As a result, it was suggested that high SDC-4 expression level of $S D C-4$ was a significant risk factor for the worse clinical outcome (HR: 13.8; 95\% CI: $1.35-84.2 ; \mathrm{p}=0.027)$ and a predictor of the clinical outcome independent of other risk factors.

\section{Discussion}

In this study, we demonstrated that $S D C-4$ expression levels in PBMCs obtained prior to WT1 vaccination 
were significantly and negatively correlated with OS of recurrent or conventional therapy-resistant GBM patients who were treated with WT1 peptide vac-

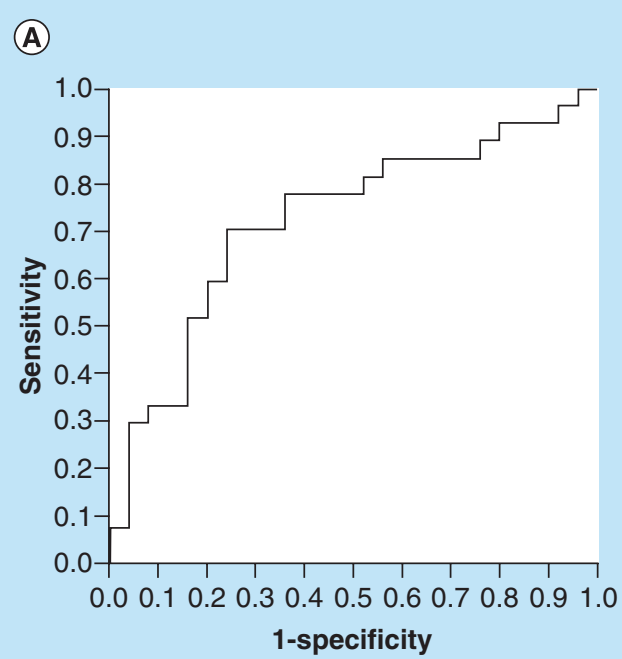

(B)

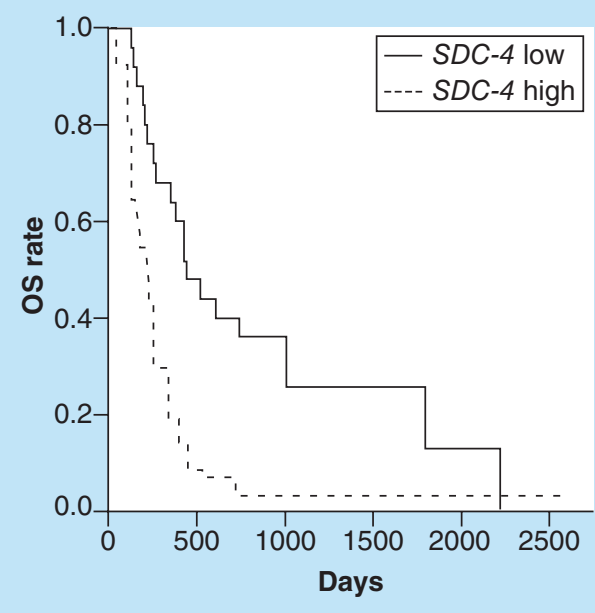

Figure 2. SDC-4 is a prediction marker for overall survival. (A) AUC-maximized receiver operating characteristic curve was generated. Optimal cutoff value, 0.001 of $S D C-4$ relative expression levels $\left(\log _{2}\left[1+2^{-\Delta C T}\right]\right)$ discriminates between long-term (OS $\geq 256$ days) and short-term (OS <256 days) survivors, and SDC-4 expression levels in long-term survivors are $\leq 0.001$. Statistical capabilities are $70.4 \%$ sensitivity, $76.0 \%$ specificity, $76.0 \%$ positive predictive value, $70.4 \%$ negative predictive value and $73.1 \%$ accuracy, and a Chi-square test shows a statistical significance $(p<0.001)$. All the 53 patients in the discovery and validation sets are included in this analysis. (B) KaplanMeier curves of OS (days) of patients with $\leq 0.001$ (SDC-4 low) and $>0.001$ (SDC-4 high) of SDC-4-expression levels are shown. Comparison of OS between the two groups is performed using a two-sided log-rank test, and the difference in OS is statistically significant $(p<0.001)$. One-year OS rates were 64.0 and $18.5 \%$ in SDC-4-low and -high patients, respectively.

OS: Overall survival. cine, and that $S D C-4$ expression levels were a useful biomarker for prediction of clinical outcome.

$\mathrm{T}$ cells are a major player in tumor immunity. $\mathrm{T}$ cells are activated through an interaction between T-cell receptors and antigen/MHC molecule complexes on antigen-presenting cells (APCs); that is regulated in a costimulatory or coinhibitory manner by accessory receptors. A costimulatory signal is transmitted by the interaction between CD28 receptor on T cells and CD80 or CD86 on APCs. On the other hand, coinhibitory signals are transmitted by a variety of molecules, including the interactions among CTLA-4 (cytotoxic T lymphocyte antigen-4) on T cells for CD80 and CD86 on APCs; programmed cell death-1 (PD-1) and its ligands PD-L1 and PD-L2; B- and T-lymphocyte attenuator and herpes virus entry mediator; Tim-3 ( $\mathrm{T}$ cell immunoglobulin- and mucin domain-containing molecules 3 ) and Tim-3L [43,44]; and by TIGIT (T cell immunoreceptor with immunoglobulin and ITIM domain) [45]. SDC-4 is a novel type of coinhibitor distinct from those listed above.

Syndecans are type-I transmembrane heparan sulfate proteoglycans that bind to the extracellular matrix and a variety of cytokines, chemokines and growth factors, thereby modifiying their local concentration, stability and accessibility to their respective receptors; consequently, they significantly influence cell proliferation and differentiation. Mammals have four known syndecans, (SDC-1, -2, -3 and -4 ), of which only SDC-4 is expressed ubiquitously; expression of the other three is tissue-restricted [46]. SDC- 4 is upregulated on activated T cells via activation of NF- $\mathrm{KB}$ [47]. In addition, it interacts with DC-associated heparan sulfate proteoglycan-dependent integrin ligand (DC-HIL) and thus mediates the coinhibitory effect of DC-HIL on T-cell activation [43]. Knockdown of SDC-4 expression enhances the T-cell response to APCs, and blockage of endogenous SDC-4 using specific antibodies or soluble SDC-4 receptor enhances T-cell reactions to syngeneic and allogeneic stimulation in vitro and exacerbated contact hypersensitivity responses in vivo [48]. Consistent with this, transplantation of SDC- $4^{-1-}$ $T$ cells into sublethally $\gamma$-irradiated allogeneic mice induces hyperproliferation of infused $\mathrm{T}$ cells [48]. Collectively, these observations indicate that SDC-4 is the T-cell ligand through which DC-HIL mediates its coinhibitory function. On the other hand, DC-HIL also binds to SDC-4 on activated T cells and is expressed most strongly by epidermal Langerhans cells, an immature type of DC [49]. DC-HIL expression levels on $\mathrm{CD}_{14}{ }^{+}$monocytes inversely correlate with allostimulatory capacity, and knockdown 
of DC-HIL enhances allostimulation. Deletion of DC-HIL abrogates the T-cell suppressor activity of myeloid-derived suppressor cells (MDSCs) [50]. These observations clearly suggest that the SDC-4/ DC-HIL pathway is one of the most important factors in regulation of immune responses mediated by $\mathrm{T}$ cells, APCs and MDSCs. Most importantly, downregulation of either or both SDC-4 and DCHIL augments T-cell-mediated-immune responses by attenuating the interaction between the two molecules and/or reducing the T-cell suppressor function of MDSCs [50].

Based on the findings described above, our present results might be interpreted as follows. Low expression of SDC-4 in PBMCs, which correlated with favorable clinical effects of WT1 peptide vaccination of GBM patients, reflected downregulated expression of SDC- 4 on T cells. In addition, this downregulation of SDC-4 augmented WT1-specific immune responses to WT1 peptide vaccination by attenuating the interaction between SDC- 4 and DC-HIL on monocytes (DCs), leading to improved clinical effects.

On the other hand, it was also reported that, besides T-cell activation state, various pathophysiological conditions, including bacterial endotoxin shock [51], acute pneumonia [52], Helicobacter pylori infection [53], atherosclerosis [54] and ischemic heart disease [55], are associated with SDC- 4 expression levels in PBMCs. Therefore, we need to examine the influence of expression levels of SDC-4, which is expressed in various types of cells, on clinical effect of WT1 peptide vaccination from multiple pathophysiological aspects. Further studies should be needed to address this issue.

Regarding the two risk factors in Table 1 and Supplementary Table 3, low-performance status is a common risk factor for poor prognosis of GBM, and the past chemotherapy might dampen immunological competence that supports WT1 peptide-based immunotherapy. On the other hand, although old age is a common risk factor for poor prognosis of GBM as well as low-performance status, no association between OS and age was detected. Since WT1 immunotherapy is a very mild therapy without significant adverse effects (only skin erythema at the vaccine injection sites) that give the organ damages, almost all patients, regardless of ages, are tolerable to the WT1 immunotherapy and may be effective to immunotherapy. On the other hand, since chemotherapy has strong adverse effects, the patients become nontolerable to it as the patients' ages increase, resulting in the decrease in clinical outcome. Therefore, age is not important prognostic factor in immunotherapy, but PS, which reflects the patients' immune conditions, is a very important prognostic factor. Repeated chemotherapy gives the damages to the immune system and thus decreases the clinical effects of the following immunotherapy. Therefore, the results that previous chemotherapy but not age is a bad prognostic factor are reasonable.

To our knowledge, only one previous study [56] involved experiments similar to ours; those authors reported biomarkers that predicted the outcome of vaccination with four kinds of HLA-A-restricted peptides in patients with conventional therapy-resistant prostate cancer. In particular, they identified LRRN3, PCDH17, HIST1H4C and PGLYRP1, but not $S D C-4$, as biomarkers that discriminated between long- ( $>900$ days) and short-term ( $\leq 900$ days) survivors. The discrepancy in the markers identified in our study and theirs, which used a different peptide vaccine, implies that different targeted TAAs lead to different immune responses in patients. This might mean that these different immune responses are dependent upon whether T cells, DCs and MDSCs or other cell types are major players in the immune reaction, and that the major players are determined by differences in the targeted TAAs and/or types of malignancies.

Since sample size of the present study was not so large, the processes to identify the $S D C-4$ as a biomarker were divided into the two: discovery and validation processes. The candidate genes identified in the discovery process using 30 patients were validated in the next process using different 23 patients in a validation set, resulting in the identification of $S D C-4$ alone as a biomarker to predict clinical outcome of WT1 peptide vaccine. We wish to perform a prospective study composed of a larger sample size in the near future, and then we may obtain a more definite finding that supports the conclusion of the present study. In addition, a large sample size-based study may give us an opportunity to find another promising biomarker for WT1 peptide vaccination, and a combination of SDC-4 and the other biomarkers may become a more precise predictor for clinical outcome of the therapy.

\section{Conclusion}

In this study, among patients with glioblastoma who received WT1 peptide vaccine, low $S D C-4$ expression level in PBMCs prior to vaccination was a prognostic factor for long-term survivors. This result was compatible with previously reported immune-suppressive functions of SDC-4 expressed on T cells. These findings suggested that $S D C-4$ might be useful not only as a biomarker to select patients who would respond to 
the immunotherapy but also as a target to improve the effect of the immunotherapy.

\section{Acknowledgements}

The authors thank the nursing teams for their care of the patients in this study, and Tomoe Umeda for coordination of clinical research.

Financial \& competing interests disclosure

The authors have no relevant affiliations or financial involvement with any organization or entity with a financial interest in or financial conflict with the subject matter or materials discussed in the manuscript. This includes employment, consultancies, honoraria, stock ownership or options, expert testimony, grants or patents received or pending, or royalties.
No writing assistance was utilized in the production of this manuscript.

Ethical conduct of research

The authors state that they have obtained appropriate institutional review board approval or have followed the principles outlined in the Declaration of Helsinki for all human or animal experimental investigations. In addition, for investigations involving human subjects, informed consent has been obtained from the participants involved.

\section{Open access}

This work is licensed under the Creative Commons Attribution 4.0 License. To view a copy of this license, visit http:// creativecommons.org/licenses/by/4.0/

\section{Executive summary}

\section{Background}

- WT1 overexpresses in leukemia and a variety of solid tumors and performs an oncogenic function, and WT1 protein is one of the most superior pan-tumor-associated antigens.

- Glioblastoma multiforme (GBM) is a malignant brain tumor with very poor prognosis, and once recurrence occurs, therapeutic options are limited.

- In 2008, we reported the promising results of a Phase II clinical study of WT1 vaccination for recurrent or conventional therapy-resistant GBM patients.

- In order to improve the clinical usefulness of WT1 peptide vaccine, it is important not only to enhance clinical efficacy but also to select the responders to WT1 peptide vaccine.

- Novel methods to predict responders prior to WT1 vaccination were awaited.

Materials \& methods

- Peripheral blood mononuclear cells from 30 GBM patients prior to WT1 vaccination in a discovery set were subjected to CDNA microarray analysis, candidate genes that differentially expressed between long- and short-term survivors were selected, followed by verification with quantitative RT-PCR.

- The filtrated candidate genes were validated for the correlation between gene expression levels and clinical effects using different 23 GBM patients of a validation set.

Results

- Thirty-two differentially expressed genes (DEGs) were extracted by CDNA microarray analysis using 30 GBM patients in a discovery set, and 15 DEGs were verified by quantitative RT-PCR.

- The 15 DEGs were validated using 23 patients in a validation set, and only SDC-4 was identified as a biomarker for prediction of overall survival (OS).

- Cut-off value of mRNA expression levels of SDC-4 that discriminated between OS of $\geq 256$ days (responders) and $O S$ of $<56$ days (nonresponders) was 0.001 , and the SDC- 4 expression levels of $\leq 0.001$ and $>0.001$ predicted responders and nonresponders, respectively, with $70.5 \%$ sensitivity and $76.0 \%$ specificity.

- One-year OS rates were 64.0 and $18.5 \%$ in SDC-4-low and -high patients, respectively.

Conclusion

- SDC-4 is a biomarker to predict clinical outcome for GBM patients treated by WT1 peptide vaccine.

\section{References}

1 Drummond IA, Madden SL, Rohwer-Nutter P, Bell GI, Sukhatme VP, Rauscher FJ 3rd. Repression of the insulinlike growth factor II gene by the Wilms tumor suppressor WT1. Science 257(5070), 674-678 (1992).

2 Hewitt SM, Hamada S, Mcdonnell TJ, Rauscher FJ 3rd, Saunders GF. Regulation of the proto-oncogenes BCL-2 and c-MYC by the Wilms' tumor suppressor gene WT1. Cancer Res. 55(22), 5386-5389 (1995).

3 Kim J, Prawitt D, Bardeesy N et al. The Wilms' tumor suppressor gene (WT1) product regulates Dax-1 gene expression during gonadal differentiation. Mol. Cell. Biol. 19(3), 2289-2299 (1999).

4 Sugiyama H. Wilms' tumor gene WT1: its oncogenic function and clinical application. Int. J. Hematol. 73(2), 177-187 (2001).

5 Nakatsuka S, Oji Y, Horiuchi T et al. Immunohistochemical detection of WT1 protein in a variety of cancer cells. Mod. Pathol. 19(6), 804-814 (2006).

6 Cheever MA, Allison JP, Ferris AS et al. The prioritization of cancer antigens: a national cancer institute pilot project for the acceleration of translational research. Clin. Cancer Res. 15(17), 5323-5337 (2009). 
7 Coosemans A, Vanderstraeten A, Tuyaerts S et al. Wilms' tumor gene 1 (WT1)-loaded dendritic cell immunotherapy in patients with uterine tumors: a Phase I/II clinical trial. Anticancer Res. 33(12), 5495-5500 (2013).

8 Van Driessche A, Van de Velde AL, Nijs G et al. Clinicalgrade manufacturing of autologous mature mRNAelectroporated dendritic cells and safety testing in acute myeloid leukemia patients in a Phase I dose-escalation clinical trial. Cytotherapy 11(5), 653-668 (2009).

9 Coosemans A, Vanderstraeten A, Tuyaerts S et al. Immunological response after WT1 mRNA-loaded dendritic cell immunotherapy in ovarian carcinoma and carcinosarcoma. Anticancer Res. 33(9), 3855-3859 (2013).

10 Izumoto S, Tsuboi A, Oka Y et al. Phase II clinical trial of Wilms tumor 1 peptide vaccination for patients with recurrent glioblastoma multiforme. J. Neurosurg. 108(5), 963-971 (2008).

11 Hashimoto N, Tsuboi A, Chiba Y et al. Immunotherapy targeting the Wilms' tumor 1 gene product for patients with malignant brain tumors. Brain Nerve 61(7), 805-814 (2009).

12 Chiba Y, Hashimoto N, Tsuboi A et al. Effects of concomitant temozolomide and radiation therapies on WT1-specific T-cells in malignant glioma. Jpn. J. Clin. Oncol. 40(5), 395-403 (2010).

13 Chiba Y, Hashimoto N, Tsuboi A et al. Prognostic value of WT1 protein expression level and MIB-1 staining index as predictor of response to WT1 immunotherapy in glioblastoma patients. Brain Tumor Pathol. 27(1), 29-34 (2010).

14 Hashimoto N, Tsuboi A, Kagawa N et al. Wilms tumor 1 peptide vaccination combined with temozolomide against newly diagnosed glioblastoma: safety and impact on immunological response. Cancer Immunol. Immunother. 64(6), 707-716 (2015).

15 Oka Y, Tsuboi A, Taguchi T et al. Induction of WT1 (Wilms' tumor gene)-specific cytotoxic T lymphocytes by WT1 peptide vaccine and the resultant cancer regression. Proc. Natl Acad. Sci. USA 101(38), 13885-13890 (2004).

16 Van Tendeloo VF, Van de Velde A, Van Driessche A et al. Induction of complete and molecular remissions in acute myeloid leukemia by Wilms' tumor 1 antigen-targeted dendritic cell vaccination. Proc. Natl Acad. Sci. USA 107(31), 13824-13829 (2010).

17 Hashii Y, Sato-Miyashita E, Matsumura R et al. WT1 peptide vaccination following allogeneic stem cell transplantation in pediatric leukemic patients with high risk for relapse: successful maintenance of durable remission. Leukemia 26(3), 530-532 (2012).

18 Tsuboi A, Oka Y, Kyo T et al. Long-term WT1 peptide vaccination for patients with acute myeloid leukemia with minimal residual disease. Leukemia 26(6), 1410-1413 (2012).

19 Oka Y, Tsuboi A, Oji Y, Kawase I, Sugiyama H. WT1 peptide vaccine for the treatment of cancer. Curr. Opin. Immunol. 20(2), 211-220 (2008).

20 Narita M, Masuko M, Kurasaki T et al. WT1 peptide vaccination in combination with imatinib therapy for a patient with CML in the chronic phase. Int. J. Med. Sci. 7(2), 72-81 (2010).

21 Oji Y, Oka Y, Nishida S et al. WT1 peptide vaccine induces reduction in minimal residual disease in an Imatinib-treated CML patient. Eur. J. Haematol. 85(4), 358-360 (2010).

22 Kawakami M, Oka Y, Tsuboi A et al. Clinical and immunologic responses to very low-dose vaccination with WT1 peptide ( 5 microg/body) in a patient with chronic myelomonocytic leukemia. Int. J. Hematol. 85(5), 426-429 (2007).

23 Ohno S, Kyo S, Myojo S et al. Wilms' tumor 1 (WT1) peptide immunotherapy for gynecological malignancy. Anticancer Res. 29(11), 4779-4784 (2009).

24 Oka Y, Tsuboi A, Murakami M et al. Wilms tumor gene peptide-based immunotherapy for patients with overt leukemia from myelodysplastic syndrome (MDS) or MDS with myelofibrosis. Int. J. Hematol. 78(1), 56-61 (2003).

25 Tsuboi A, Oka Y, Nakajima $\mathrm{H}$ et al. Wilms tumor gene WT1 peptide-based immunotherapy induced a minimal response in a patient with advanced therapy-resistant multiple myeloma. Int. J. Hematol. 86(5), 414-417 (2007).

26 Nishioka M, Tanemura A, Nishida S et al. Vaccination with WT-1 (Wilms' tumor gene-1) peptide and BCG-CWS in melanoma. Eur. J. Dermatol. 22(2), 258-259 (2012).

27 Ohta H, Hashii Y, Yoneda A et al. WT1 (Wilms tumor 1) peptide immunotherapy for childhood rhabdomyosarcoma: a case report. Pediatr. Hematol. Oncol. 26(1), 74-83 (2009).

28 Tsuboi A, Oka Y, Osaki T et al. WT1 peptide-based immunotherapy for patients with lung cancer: report of two cases. Microbiol. Immunol. 48(3), 175-184 (2004).

29 Takahara A, Koido S, Ito M et al. Gemcitabine enhances Wilms' tumor gene WT1 expression and sensitizes human pancreatic cancer cells with WT1-specific T-cellmediated antitumor immune response. Cancer Immunol. Immunother. 60(9), 1289-1297 (2011).

30 Nishida S, Koido S, Takeda Y et al. Wilms tumor gene (WT1) peptide-based cancer vaccine combined with gemcitabine for patients with advanced pancreatic cancer. J. Immunother. 37(2), 105-114 (2014).

31 Dohi S, Ohno S, Ohno Y et al. WT1 peptide vaccine stabilized intractable ovarian cancer patient for one year: a case report. Anticancer Res. 31(7), 2441-2445 (2011).

32 Miyatake T, Ueda Y, Morimoto A et al. WT1 peptide immunotherapy for gynecologic malignancies resistant to conventional therapies: a Phase II trial. J. Cancer Res. Clin. Oncol. 139(3), 457-463 (2013).

33 Ohno S, Dohi S, Ohno Y et al. Immunohistochemical detection of WT1 protein in endometrial cancer. Anticancer Res. 29(5), 1691-1695 (2009).

34 Sasabe E, Hamada F, Iiyama T, Udaka K, Sugiyama $\mathrm{H}$, Yamamoto T. Wilm's tumor gene WT1 peptide immunotherapy for pulmonary metastasis from adenoid cystic carcinoma of the salivary gland. Oral Oncol. 47(1), 77-78 (2011).

35 Shirakata T, Oka Y, Nishida S et al. WT1 peptide therapy for a patient with chemotherapy-resistant salivary gland cancer. Anticancer Res. 32(3), 1081-1085 (2012). 
36 Ostrom QT, Bauchet L, Davis FG et al. The epidemiology of glioma in adults: a "state of the science" review. Neuro Oncol. 16(7), 896-913 (2014).

37 Ogi C, Aruga A. New concepts of biomarkers and clinical outcomes for therapeutic cancer vaccines in clinical trials. Immunotherapy 6(10), 1025-1036 (2014).

38 Shippy R, Fulmer-Smentek S, Jensen RV et al. Using RNA sample titrations to assess microarray platform performance and normalization techniques. Nat. Biotechnol. 24(9), 1123-1131 (2006)

39 Allison DB, Cui X, Page GP, Sabripour M. Microarray data analysis: from disarray to consolidation and consensus. Nat. Rev. Genet. 7(1), 55-65 (2006).

40 Shi L, Reid LH, Jones WD et al. The MicroArray Quality Control (MAQC) project shows inter- and intraplatform reproducibility of gene expression measurements. Nat. Biotechnol. 24(9), 1151-1161 (2006).

41 NCBI nucleotide database. www.ncbi.nlm.nih.gov/nuccore

42 The R Project for Statistical Computing. www.r-project.org

43 Chung JS, Dougherty I, Cruz PD Jr, Ariizumi K. Syndecan-4 mediates the coinhibitory function of DC-HIL on T cell activation. J. Immunol. 179(9), 5778-5784 (2007).

44 Chung JS, Sato K, Dougherty Ii, Cruz PD Jr, Ariizumi K. DC-HIL is a negative regulator of T lymphocyte activation. Blood 109(10), 4320-4327 (2007).

45 Yu X, Harden K, Gonzalez LC et al. The surface protein TIGIT suppresses $\mathrm{T}$ cell activation by promoting the generation of mature immunoregulatory dendritic cells. Nat. Immunol. 10(1), 48-57 (2009).

46 Teixe T, Nieto-Blanco P, Vilella R, Engel P, Reina M, Espel E. Syndecan-2 and -4 expressed on activated primary human CD4+ lymphocytes can regulate T cell activation. Mol. Immunol. 45(10), 2905-2919 (2008).

47 Okuyama E, Suzuki A, Murata M et al. Molecular mechanisms of syndecan-4 upregulation by TNF-alpha in the endothelium-like EAhy926 cells. J. Biochem. 154(1), 41-50 (2013).

48 Chung JS, Tomihari M, Tamura K, Kojima T, Cruz PD Jr, Ariizumi K. The DC-HIL ligand syndecan- 4 is a negative regulator of T-cell allo-reactivity responsible for graft-versushost disease. Immunology 138(2), 173-182 (2013).

49 Chung JS, Yudate T, Tomihari M, Akiyoshi H, Cruz PD Jr, Ariizumi K. Binding of DC-HIL to dermatophytic fungi induces tyrosine phosphorylation and potentiates antigen presenting cell function. J. Immunol. 183(8), 5190-5198 (2009).

50 Chung JS, Tamura K, Akiyoshi H, Cruz PD Jr, Ariizumi K. The DC-HIL/syndecan-4 pathway regulates autoimmune responses through myeloid-derived suppressor cells. J. Immunol. 192(6), 2576-2584 (2014).

51 Ishiguro K, Kadomatsu K, Kojima T et al. Syndecan-4 deficiency leads to high mortality of lipopolysaccharideinjected mice. J. Biol. Chem. 276(50), 47483-47488 (2001).

52 Tanino Y, Chang MY, Wang X et al. Syndecan-4 regulates early neutrophil migration and pulmonary inflammation in response to lipopolysaccharide. Am. J. Respir. Cell Mol. Biol. 47(2), 196-202 (2012).

53 Smith MF Jr, Novotny J, Carl VS, Comeau LD. Helicobacter pylori and toll-like receptor agonists induce syndecan- 4 expression in an NF-kappaB-dependent manner. Glycobiology 16(3), 221-229 (2006).

54 Boyanovsky BB, Shridas P, Simons M, Van der Westhuyzen DR, Webb NR. Syndecan-4 mediates macrophage uptake of group V secretory phospholipase A2-modified LDL. J. Lipid Res. 50(4), 641-650 (2009).

55 Li J, Brown LF, Laham RJ, Volk R, Simons M. Macrophagedependent regulation of syndecan gene expression. Circ. Res. 81(5), 785-796 (1997).

56 Komatsu N, Matsueda S, Tashiro K et al. Gene expression profiles in peripheral blood as a biomarker in cancer patients receiving peptide vaccination. Cancer 118(12), 3208-3221 (2012). 


\section{Affiliations}

\section{Satoshi Takashima}

Department of Respiratory Medicine, Allergy \& Rheumatic Diseases, Osaka University Graduate School of Medicine, 2-2 Yamadaoka, Suita City, Osaka 565-0871, Japan

\section{Yoshihiro Oka}

Department of Respiratory Medicine, Allergy \& Rheumatic Diseases, Osaka University Graduate School of Medicine, 2-2 Yamadaoka, Suita City, Osaka 565-0871, Japan

and

Department of Cancer Immunology, Osaka University Graduate School of Medicine, 1-7 Yamadaoka, Suita City, Osaka 565-0871, Japan

and

Department of Immunopathology, Immunology Frontier Research Center (World Premier International Research Center), Osaka University, 3-1 Yamadaoka, Suita City, Osaka 565-0871, Japan

\section{Fumihiro Fujiki}

Department of Cancer Immunology, Osaka University Graduate School of Medicine, 1-7 Yamadaoka, Suita City, Osaka 565-0871, Japan

\section{Soyoko Morimoto}

Department of Cancer Immunology, Osaka University Graduate School of Medicine, 1-7 Yamadaoka, Suita City, Osaka 565-0871, Japan

\section{Hiroko Nakajima}

Department of Cancer Immunology, Osaka University Graduate School of Medicine, 1-7 Yamadaoka, Suita City, Osaka 565-0871, Japan

\section{Yoshiki Nakae}

Department of Respiratory Medicine, Allergy \& Rheumatic Diseases, Osaka University Graduate School of Medicine, 2-2 Yamadaoka, Suita City, Osaka 565-0871, Japan and

Department of Hematology, Kitano Hospital, 2-4-10 Ohgimachi, Kitaku, Osaka City, Osaka 530-8480, Japan

\section{Jun Nakata}

Department of Cancer Immunotherapy, Osaka University Graduate School of Medicine, 2-2 Yamadaoka, Suita City, Osaka 565-0871, Japan

\section{Sumiyuki Nishida}

Department of Respiratory Medicine, Allergy \& Rheumatic Diseases, Osaka University Graduate School of Medicine, 2-2 Yamadaoka, Suita City, Osaka 565-0871, Japan

\section{Naoki Hosen}

Department of Cancer Stem Cell Biology, Osaka University Graduate School of Medicine, 1-7 Yamadaoka, Suita City, Osaka 565-0871, Japan

\section{Naoya Tatsumi}

Department of Cancer Immunotherapy, Osaka University Graduate School of Medicine, 2-2 Yamadaoka, Suita City, Osaka 565-0871, Japan

\section{Kenji Mizuguchi}

National Institutes of Biomedical Innovation, Health \& Nutrition 7-6-8 Saito-Asagi, Ibaraki City, Osaka 567-0085, Japan

\section{Naoya Hashimoto}

Department of Neurosurgery, Kyoto Prefectural University Graduate Schoolof Medicine, 465 Kajii-cho, Hirokoji-agaru, Kawaramachi-dori, Kamigyo-ku, Kyoto City, Kyoto 602-8566, Japan and

Department of Neurosurgery, Graduate School of Medicine, Osaka University, 2-2 Yamadaoka,Suita City, Osaka 565-0871, Japan

\section{Yusuke Oji}

Department of Cancer Stem Cell Biology, Osaka University Graduate School of Medicine, 1-7 Yamadaoka, Suita City, Osaka 565-0871, Japan

\section{Akihiro Tsuboi}

Department of Cancer Immunotherapy, Osaka University Graduate School of Medicine, 2-2 Yamadaoka, Suita City, Osaka 565-0871, Japan

\section{Atsushi Kumanogoh}

Department of Respiratory Medicine, Allergy \& Rheumatic Diseases, Osaka University Graduate School of Medicine, 2-2 Yamadaoka, Suita City, Osaka 565-0871, Japan and Department of Immunopathology, Immunology Frontier Research Center (World Premier International Research Center), Osaka University, 3-1 Yamadaoka, Suita City, Osaka 565-0871, Japan

\section{Haruo Sugiyama}

Department of Cancer Immunology, Osaka University Graduate School of Medicine, 1-7 Yamadaoka, Suita City, Osaka 565-0871, Japan 